\title{
Fer romagnetism in fcc Twinned 2.4 nm Size Pd Nanoparticles
}

\author{
B. Sampedro, ${ }^{1,2}$ P. Crespo, ${ }^{1,2}$ A. Hernando, ${ }^{1,2}$ R. Litrán, ${ }^{3}$ J. C. Sánchez López, ${ }^{3}$ C. López Cartes, ${ }^{3}$ A. Fernandez, ${ }^{3}$ \\ J. Ramírez, ${ }^{4}$ J. González Calbet, ${ }^{1,4}$ and M. Vallet ${ }^{1,5}$ \\ ${ }^{1}$ Instituto de Magnetismo Aplicado, "Salvador Velayos," UCM, RENFE, CSIC, Las Rozas, P.O. Box 155, Madrid 28230, Spain \\ ${ }^{2}$ Departamento de Física de Materiales, UCM, Madrid 28040, Spain \\ ${ }^{3}$ Instituto de Ciencias de Materiales de Sevilla (ICMSE), Centro Mixto CSIC-UNIV, Sevilla 41092, Spain \\ ${ }^{4}$ Departamento de Química Inorgánica, Facultad de Química, UCM, Madrid 28040, Spain \\ ${ }^{5}$ Departamento de Química Inorgánica, Facultad de Farmacia, UCM, Madrid 28040, Spain
}

(Received 24 July 2003; published 4 December 2003)

\begin{abstract}
The onset of ferromagnetism has been experimentally observed in small Pd particles of average diameter $2.4 \mathrm{~nm}$. High-resolution studies reveal that a high percentage of the fcc particle exhibits single and multiple twinning boundaries. The spontaneous magnetization close to $0.02 \mathrm{emu} / \mathrm{g}$ seems to indicate that only a small fraction of atoms holds a permanent magnetic moment and contributes to ferromagnetism. The possible origin of ferromagnetism is briefly discussed according to different models recently reported.
\end{abstract}

DOI: 10.1103/PhysRevLett.91.237203

PACS numbers: 75.50.Cc, 75.50.Tt, 75.70.Cn

The possible ferromagnetism of $\mathrm{Pd}$ and late $4 d$ transition metals has been intensively investigated giving rise to a continuous issue of theoretical and experimental studies. A free $\mathrm{Pd}$ atom has a $[\mathrm{Kr}] 4 d^{10}$ configuration and is not magnetic. The crystalline structure of Pd metal is face-centered cubic (fcc) with a lattice constant of $a=3.8898 \AA$. The $d$ band is almost filled and the $s$ band partially filled, giving rise to a configuration designed formally as $(4 d)^{10-\xi}(5 s)^{\xi}$, where $\xi \approx 0$. Pd metal exhibits enhanced Pauli paramagnetism with large susceptibility. However, in bulk Pd no spontaneous ferromagnetic order has been observed. Although the density of states, $N(E)$, shows a sharp peak $\left[N\left(E_{F}\right)=\right.$ 1.23 states $\mathrm{eV}^{-1} \operatorname{spin}^{-1}$ atom ${ }^{-1}$ ] [1] just below the Fermi level $E_{F}$, the Stoner criterion for ferromagnetism, $N\left(E_{F}\right) I>1$, is not satisfied ( $I$ stands for the Stoner parameter typically $0.71 \mathrm{eV}$ for Pd) [1]. Nevertheless, Pd lies close to a ferromagnetic instability.

The factors that can affect the onset of ferromagnetism are those increasing $N\left(E_{F}\right)$ : i.e., (i) confinement effects associated with a reduced coordination number that have been studied by analyzing thin layers [2] and clusters formed by a few atoms, (ii) local symmetry changes analyzed by observing the effect of vacancies [1] or by increasing the percentage of atoms at the grain boundaries or interfaces, and (iii) lattice expansion that induces a narrowing of the $d$ band that according to theoretical calculations should be of the order of $5.3 \%$ for the onset of ferromagnetism [3].

Experiments carried out by Taniyama et al. [4] in 1997 have evidenced the appearance of a magnetic moment in $\mathrm{Pd}$ clusters with an average radius below $7 \mathrm{~nm}$. Furthermore, these authors found ferromagnetic behavior of Pd particles of $5.9 \mathrm{~nm}$ average radius with an atomic moment of $0.23 \mu_{B}$ per surface atom. Since the lattice constant observed in these particles was of $3.9 \AA$ lattice expansion can be reasonably disregarded as the origin of ferromagnetism. It has been theoretically estimated [5] that as a consequence of the strong surface energy anisotropy clusters of Pd exhibit a noncrystallographic icosahedral instead of the typical bulk fcc symmetry. Recent experimental observations [6] have shown the coexistence of several structures in Pd nanoparticles as fcc cubo-octahedron, icosahedrons, truncated decahedrons, and single and multiple-twinned fcc configurations. The important point is that under noncubic local field symmetry the $d$ atomic level does not split in two, $e_{g}$ and $t_{2 g}$, sublevels giving rise to a drastic narrowing of the $4 d$ band sufficient for the Stoner criterion to be held. This is the case for icosahedral configurations where ferromagnetic behavior of Pd was predicted theoretically by Reddy et al. [7] and Vitos et al. [5].

In fact, according to the measurements performed by Cox et al. [8], no evidence of ferromagnetism was observed on 13-150 atom Pd clusters by using SternGuerlach experiments. However, Taniyama et al. [4] found, by using a SQUID magnetometer, an increase of the ferromagnetic susceptibility by decreasing the particle size from 9.9 down to $5.9 \mathrm{~nm}$ median radius. Unfortuantely the structure of these Pd nanoparticles was not described.

In this Letter we report on ferromagnetism observed in fcc Pd nanoparticles with $1.2 \mathrm{~nm}$ of median radius and with an average number of 530 atoms per particle. It is to be noted that high-resolution observations show that a high percentage of these particles exhibit single or multiple-twinned structures.

Stabilized palladium nanoparticles have been obtained by the redox-controlled size-selective method, using $R_{4} N^{+} X^{-}$as the surfactant in tetrahydrofurane. A solution of $\operatorname{Pd}\left(\mathrm{NO}_{3}\right)_{2}$ from Aldrich was used as the palladium precursor in an excess of tetrabutylammonium acetate $\left(n-\mathrm{C}_{4} \mathrm{H}_{9}\right)_{4} \mathrm{~N}^{+}\left(\mathrm{CH}_{3} \mathrm{CO}_{2}^{-}\right)$. Nitrate is displaced by the more effective acetate ligand, which is followed by the 


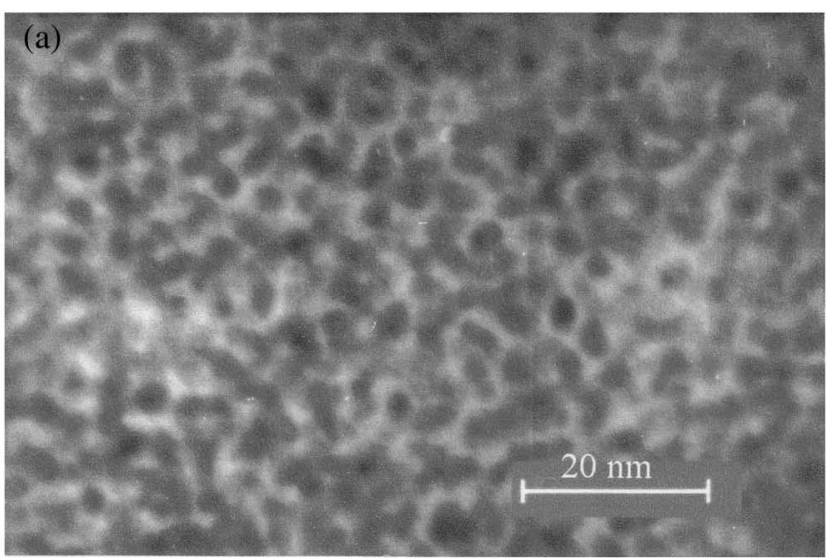

(b)

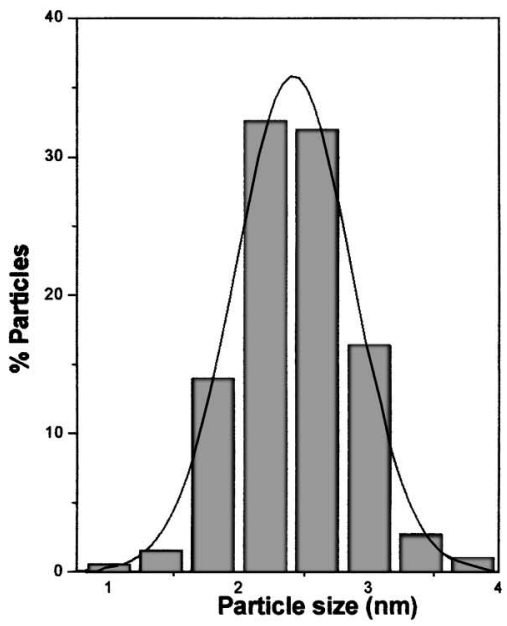

FIG. 1. (a) Transmission electron micrographs of colloidal Pd stabilized by tetrabutylammonium acetate. (b) Particle sizedistribution histogram from the micrograph shown in (a). The number of analyzed particles has been $N=405$, the mean diameter, $d=2.4 \mathrm{~nm}$, and the standard deviation, $s=0.3 \mathrm{~nm}$.

reduction of the $\mathrm{Pd}^{2+}$ by electron transfer giving rise to $\mathrm{Pd}^{0}$-stabilized clusters [9]. Precursor salts are high-purity Aldrich products, in which no evidences of $3 d$ or $4 d$ transition elements have been detected. Chemical reac- tions have been carried out under inert gas flow, in order to avoid oxygen contamination.

Figures 1(a) and 1(b) show a general view of the palladium nanoparticles and its corresponding particle size distribution, respectively. The average mean particle size was estimated to be $2.4 \mathrm{~nm}$ by transmission electron microscopy (TEM) what corresponds to an average number of atoms per particle of ca. 530. The estimated number of atoms at the surface or with reduced coordination numbers represents around $46 \%$ of the total number of atoms in the nanoparticles. The tretaalkylammonium chains, which act simultaneously as the reductant and the protective agent, maintain the nanoparticles separated in the assembly. The Pd colloids are likely stabilized through electrostatic interactions between the $R_{4} N^{+} X^{-}$ dipoles and the palladium surface [10].

Structures of Pd nanoparticles were characterized by using a JEOL 300 FEG high-resolution electron microscope, HRTEM, with resolution 1.6 ̊. Fourier filtration has been performed by windowing the Fourier transform (FT) around amplitude maxima (spots) representing the lattice periodicities. Although Fourier filtering may introduce "ghost" lattices, it is a powerful tool to underline the structural defects in a crystalline area. The filtration technique applied is based on the fact that all the information concerning a periodical structure is concentrated in the maxima of its Fourier transform and the noise is randomly distributed in Fourier space. We can isolate the information from the periodical structure by using a circular mask (window) that selects the sharp maxima and by performing an inverse Fourier transform which reconstructs the original image without noise.

Figure 2(a) corresponds to a Fourier filtered HRTEM image obtained in a particle with $2 \mathrm{~nm}$ in diameter that shows fcc structure. A twin boundary is evident. Figure 2(b) shows the corresponding FT diffraction pattern, where splitting of the spots due to the twinning is clearly appreciated. According to particle size distribution, most of the particles observed present diameter
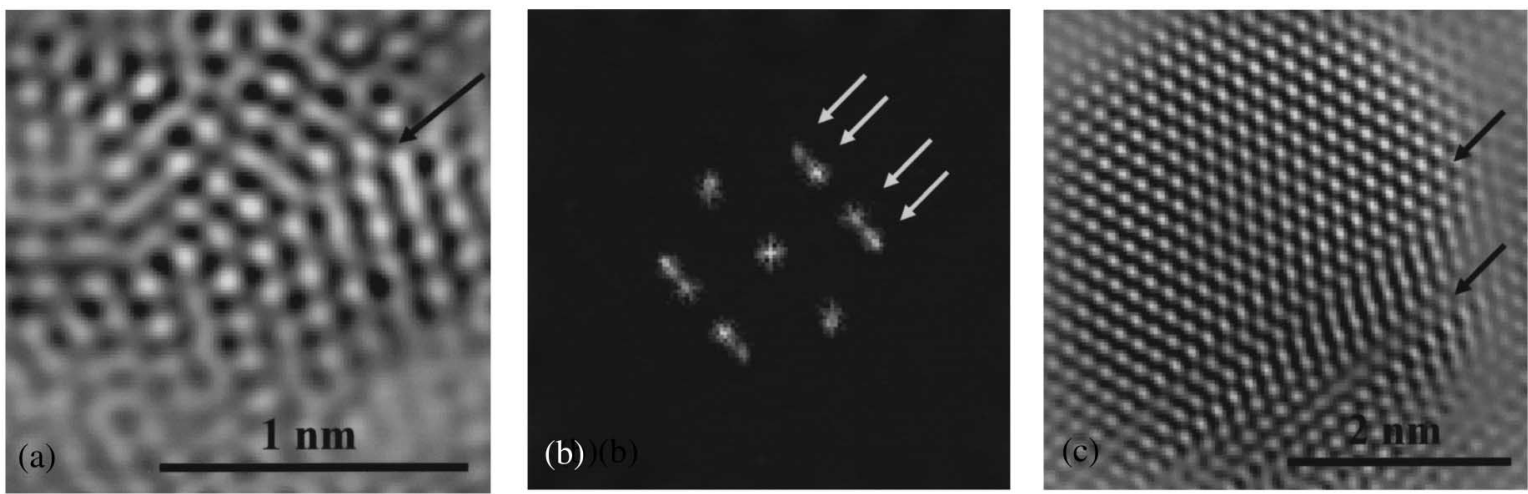

FIG. 2. (a) Fourier filtered HRTEM image of a Pd nanoparticle (diameter $=2 \mathrm{~nm}$ ). The twin boundary is arrowed. (b) Corresponding FT diffraction pattern along the [111] direction. The splitting of the FT spots due to twinning is indicated. (c) Fourier filtered HRTEM image of a Pd nanocrystal (diameter $=4.4 \mathrm{~nm}$ ) showing two twin boundaries. 
around $2.4 \mathrm{~nm}$. However, a small amount of particles shows diameter as large as 4-4.5 nm [see Fig. 1(b)]. Also some of these largest particles are twinned. An example is shown in Fig. 2(c) where two twin boundaries are apparent.

Magnetic measurements have been performed using a quantum design SQUID magnetometer. Diamagnetic contributions corresponding to the sample holder system have been previously measured. After removing this contribution from the total magnetization that one corresponding to Pd can be directly obtained.

Ferromagnetic ordering in Pd nanoparticles finds definitive evidence in the hysteresis loops shown in Fig. 3. A clear ferromagnetic behavior, with Curie temperature above $300 \mathrm{~K}$, is observed. From the shape of the hysteresis loops obtained at different temperatures between 5 and $275 \mathrm{~K}$ it is inferred that particles with blocked magnetization contribute to the magnetization process. Coercivity decreases with temperature roughly linearly from $100 \mathrm{Oe}$ at zero Kelvin down to zero at $340 \mathrm{~K}$. Hence, $340 \mathrm{~K}$ should rather correspond to a blocking temperature $T_{b}$. Through the relation $T_{b}=k v / 25 k_{B}\left(k_{B}\right.$ stands for the Boltzmann constant and $v=7 \times 10^{-27} \mathrm{~m}^{3}$ the particle average volume) the effective anisotropy $k$ of Pd particles is estimated to be $k=2 \times 10^{7} \mathrm{Jm}^{-3}$. This is a quite reasonable value if we consider that the main contribution to the anisotropy of these approximately spherical particles is the crystalline anisotropy. The relative large atomic weight of Pd, when compared to $3 d$ transition ferromagnetic elements, accounts for the relative enhancement of the spin orbit coupling strength and consequently for the increase of the anisotropy constant. The magnetization reached at the high field branch of the loop is far from saturation as is probably due to the coexistence of blocked ferromagnetic entities with superparamagnetic particles and paramagnetic atoms.

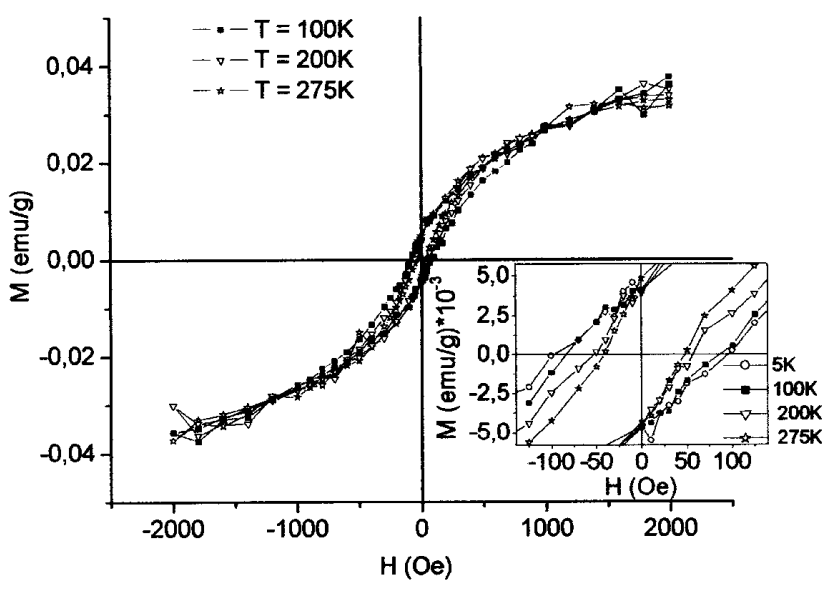

FIG. 3. Hysteresis loops at different temperatures of Pd nanoparticles. Inset: a more detailed view of the thermal coercivity response of the same sample.
Figure 4 describes the thermal dependence of magnetization under a dc applied field of $1 \mathrm{kOe}$ for $2.4 \mathrm{~nm}$ average mean particle size. The corresponding curve obtained in bulk Pd metal is also shown.

By extrapolating at zero field magnetization in Fig. 3, a value close to $0.02 \mathrm{emu} / \mathrm{g}$ is obtained. If the particles are assumed to be randomly distributed in orientation the estimated value for the permanent magnetic moment is $0.04 \mathrm{emu} / \mathrm{g}$ for uniaxial anisotropy or $0.025 \mathrm{emu} / \mathrm{g}$ for cubic symmetry. The permanent moment is likely associated with an unknown fraction of the total number of nanoparticles and furthermore with an unknown fraction of the total number of atoms (surface or twinned boundary regions) at each of these nanoparticles. Hence, the experiments can also supply a hint about the magnetic moment per atom if we consider it uniformly distributed over all the particles and over all the atoms of each particle that approximately yields $10^{-3} \mathrm{Bohr}$ magnetons per atom. This value is 1 order of magnitude smaller than that experimentally found by Taniyama et al. [4], and 2 orders below that theoretically predicted for icosahedral structure [5]. It seems to indicate that probably only a very small fraction of the total number of atoms exhibits permanent magnetic moment and eventually ferromagnetism.

The analysis by SQUID magnetometry of the palladium precursor salt shows a diamagnetic behavior. The presence of impurities has not been detected by $\mathrm{x}$ ray dispersive energy analysis and electron probe microanalysis; therefore, possible impurities of $3 d$ transition elements or other $4 d$ impurities content should be much lower than 0.1 at $\%$ that is the content required to account for the observed magnetic moment per atom. Therefore,

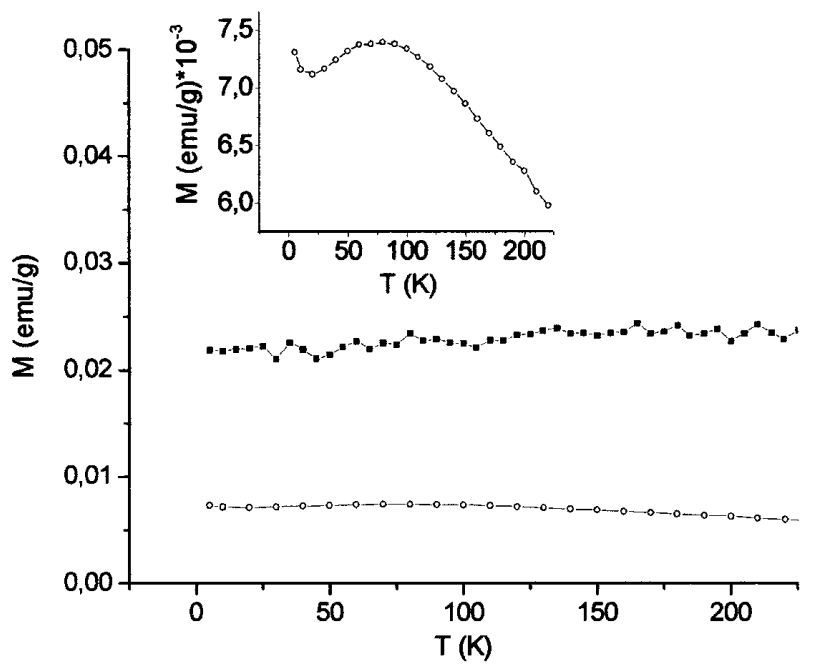

FIG. 4. Thermal dependence of magnetization under a dc applied field of $1 \mathrm{kOe}$ for $\mathrm{Pd} 2.4 \mathrm{~nm}$ average mean particle size (solid squares) and bulk Pd (open circles). Inset: detailed thermal dependence for Pd bulk metal measured under the same applied field but plotted in a different scale. 
impurities can be disregarded as a possible origin of the observed magnetic behavior.

Since surface atoms are located in a non-fcc symmetry, an extremely high percentage (46 at. \%) of them, characteristic of small size clusters, could be invoked as a possible cause of the onset of ferromagnetism. The eventual increase of $N\left(E_{F}\right)$ at the cluster surface due to the enhancement of electron localization as well as the effect produced by the electric interaction with the surfactant molecules may contribute to the appearance of permanent magnetic moment and high local magnetic anisotropy [11]. If the surface atoms were considered responsible for ferromagnetism the experimental values would lead approximately to a very weak contribution of $5 \times 10^{-3}$ Bohr magnetons per atom. Moreover, first principle calculations predict that fcc single crystals Pd clusters with close packed (111), (100), and (110) facets [5] are not ferromagnetic.

Local deviation from cubic symmetry near the twin boundaries could also be invoked to account for the ferromagnetic behavior [5]. The strong surface energy anisotropy acts as the driving force for twinning in small Pd clusters. Therefore, the twinning boundary density is expected, and experimentally observed in this Letter and in a previous paper [6], to be noticeably enhanced in small clusters. Consequently, we suggest that the onset of ferromagnetism, experimentally observed and reported here, may be associated with the small fraction of atoms located in the vicinity of the twin boundary, where the increase of $N\left(E_{F}\right)$ promotes the induction of a spontaneous magnetic moment.

In conclusion, we have observed the onset of ferromagnetism in fcc twinned $2.4 \mathrm{~nm}$ Pd nanoparticles.

[1] N. Takano, T. Kai, K. Shiiki, and F. Terasaki, Solid State Commun. 97, 153 (1996).

[2] R. Pfandzelter, G. Steierl, and C. Rau, Phys. Rev. Lett. 74, 3467 (1995); S. Blügel, Phys. Rev. B 51, 2025 (1995).

[3] H. Chen, N. E. Brener, and J. Callaway, Phys. Rev. B 40, 1443 (1989); V. L. Moruzzi and P. M. Marcus, Phys. Rev. B 39, 471 (1989).

[4] T. Taniyama, E. Ohta, and T. Sato, Europhys. Lett. 38, 195 (1997).

[5] L. Vitos and B. Johansson, Phys. Rev. B 62, 11 957(R) (2000).

[6] M. José-Yacamán, M. Marín-Almazo, and J. A. Ascencio, J. Mol. Catal. A: Chem. 173, 61 (2001).

[7] B. V. Reddy, S. N. Khanna, and B. I. Dunlap, Phys. Rev. Lett. 70, 3323 (1993); electron localization in twin boundaries of $\mathrm{Ni}$ was previously calculated by F. Yndurain and L. M. Falicov, Phys. Rev. Lett. 37, 928 (1976).

[8] A. J. Cox, J. G. Louderback, S. E. Aspel, and L. A. Bloomfield, Phys. Rev. B 49, 12295 (1994).

[9] M.T. Reetz and M. Maase, Adv. Mater. 11, 773 (1999).

[10] M. Reetz, W. Helbig, S. A. Quaiser, U. Stimming, N. Breuer, and R. Vogel, Science 267, 367 (1995).

[11] P. Gambardella et al., Science 300, 1130 (2003). 